Commun.Fac.Sci.Univ.Ank.Series A 1

Volume 58, Number 1, Pages 23-28 (2009)

ISSN $1303-5991$

\title{
HOMOTHETIC MOTIONS AND BICOMPLEX NUMBERS
}

\author{
FAIK BABADAĞ, YUSUF YAYLI AND NEJAT EKMEKCI
}

\begin{abstract}
In this study, one of the concepts of conjugate which is defined [1] for bicomplex numbers is investigated. In this case, the metric, in four dimensional semi-Euclidean space $\mathrm{E}_{2}^{4}$, has been defined by the help of the concept of the conjugate. We define a motion in $\mathrm{E}_{2}^{4}$ with the help of the metric in bicomplex numbers. We show that the motions defined by a curve lying on a hypersurface $\mathrm{M}$ of $\mathrm{E}_{2}^{4}$ are homothetic motions . Furthermore, it is shown that the motion defined by a regular curve of order $\mathrm{r}$ and derivations of the curve on the hypersurface $\mathrm{M}$ has only one acceleration centre of order $(\mathrm{r}-1)$ at every t- instant.
\end{abstract}

\section{INTRODUCTION}

In 2006, Dominic Rochon and S.Tremblay, presented a paper based on bicomplex quantum mechanics : II. The Hilbert Space [1,2]. Bicomplex (hyperbolic) numbers are given in this paper from a number of different points of view of Hilbert Space for quantum mechanics.

In this study, a new operator similar to Hamilton operator [3] has been given for bicomplex numbers [4], homothetic motion has been defined by the help of the components of the hyper surface and different theorems have been given . It is shown that this study can be repeated for bicomplex numbers,which is a homothetic motion in four-dimensional semi-Euclidean spaces and this homothetic motion satisfies all of the properties [5].

\section{Bicomplex Numbers}

Bicomplex numbers are defined by $[1,2,6]$

$$
T=\left\{z_{1}+z_{2} i_{2}: z_{1}, z_{2} \in C\left(i_{1}\right)\right\}
$$

where the imaginary units $i_{1}, i_{2}$ and $j$ are governed by the rules:

$$
i_{1}^{2}=i_{2}^{2}=-1, \quad j^{2}=1
$$

Received by the editors March 16, 2009, Accepted: June. 05, 2009.

2000 Mathematics Subject Classification. 53A05, 53A17.

Key words and phrases. Bicomplex number, Homothetic motion, Hypersurface, Pole points, Semiorthogonal matrix. 


$$
i_{1} \cdot i_{2}=i_{2} . i_{1}=j: i_{1} \cdot j=j \cdot i_{1}=-i_{2}: i_{2} \cdot j=j \cdot i_{2}=-i_{1}
$$

where we define $C\left(i_{k}\right)=\left\{x+y i_{k}: i_{k}^{2}=-1\right.$ and $\left.x, y \in R\right\}$ for $k=1,2$. Hence it is easy to see that the multiplication of two bicomplex numbers is commutative . It is also convenient to write the set of bicomplex numbers as

$$
T=\left\{w\left|w=w_{1}+w_{2} i_{1}+w_{3} i_{2}+w_{4} j \quad\right|\left(w_{1}, w_{2}, w_{3}, w_{4}\right) \in R .\right\}
$$

Complex conjugation plays an important role both for algebraic and geometric properties of complex numbers $[1,2]$.

$$
\begin{aligned}
\bar{w} & =\overline{z_{1}+z_{2} i_{2}}=z_{1}-z_{2} i_{2} \\
& =w_{1}+w_{2} i_{1}-w_{3} i_{2}-w_{4} j
\end{aligned}
$$

where $\bar{w} \cdot w=w_{1}^{2}-w_{2}^{2}+w_{3}^{2}-w_{4}^{2}+2 i_{2}\left(w_{1} w_{2}+w_{3} w_{4}\right)$ and $w_{1} w_{2}+w_{3} w_{4}=0$ i.e

$$
\bar{w} \cdot w=w_{1}^{2}-w_{2}^{2}+w_{3}^{2}-w_{4}^{2} \in R
$$

The system $\{T, \oplus, R,+, ., \circ, \otimes\}$ is a commutative algebra. It is referred as the bicomplex number algebra and shown with $T$, briefly one of the bases of this algebra is $\left\{1, i_{1}, i_{2}, j\right\}$ and the dimension is 4 .

2.1. Multiplication Operation. The operation

$$
\otimes: \begin{aligned}
T \times T & \rightarrow T \\
(u, w) & \rightarrow u \otimes w=w \otimes u
\end{aligned}
$$

is defined with the following multiplication

$$
\begin{aligned}
u \otimes w= & \left(u_{1}+i_{1} u_{2}+i_{2} u_{3}+j u_{4}\right) \otimes\left(w_{1}+i_{1} w_{2}+i_{2} w_{3}+j w_{4}\right) \\
= & \left(u_{1} w_{1}-u_{2} w_{2}-u_{3} w_{3}+u_{4} w_{4}\right)+i_{1}\left(u_{1} w_{2}+u_{2} w_{1}-u_{3} w_{4}-u_{4} w_{3}\right) \\
& +i_{2}\left(u_{1} w_{3}-u_{2} w_{4}+u_{3} w_{1}-u_{4} w_{2}\right)+j\left(u_{1} w_{4}+u_{2} w_{3}+u_{3} w_{2}+u_{4} w_{1}\right)
\end{aligned}
$$

It is possible to give the production in $T$ similar to the Hamilton operators which has been given in [3]. Because it is not a quaternion commutative matrix, there are two different matrixes for each of the right and left-multiplications. However, here only one matrix is obtained. Because it is similar to Hamilton operators. (for Hamilton operators see $[3,5]$ ). If $w=w_{1}+w_{2} i_{1}+w_{3} i_{2}+w_{4} j$ is a bicomplex number , then $N^{+}=N^{-}=N$ is defined as

$$
N(w)=\left[\begin{array}{rrrr}
w_{1} & -w_{2} & -w_{3} & w_{4} \\
w_{2} & w_{1} & -w_{4} & -w_{3} \\
w_{3} & -w_{4} & w_{1} & -w_{2} \\
w_{4} & w_{3} & w_{2} & w_{1}
\end{array}\right]
$$

If $w=z_{1}+z_{2} i_{2}$ then

$$
N(w)=\left[\begin{array}{cc}
N\left(z_{1}\right) & -N\left(z_{2}\right) \\
N\left(z_{2}\right) & N\left(z_{1}\right)
\end{array}\right]
$$


Using the definition of $N$, the multiplication of two bicomplex numbers $x$ and $y$ is given by

$$
w \otimes u=N(w) \cdot u \quad: u, w \in T
$$

and

$$
\operatorname{det} N(w)=\left[w_{1}^{2}-w_{2}^{2}+w_{3}^{2}-w_{4}^{2}+2\left(w_{1} w_{2}+w_{3} w_{4}\right)\right]^{2}
$$

\section{Homothetic Motions At $\mathrm{E}_{2}^{4}$}

Let,

$$
\begin{aligned}
M & =\left\{w=\left(w_{1}, w_{2}, w_{3}, w_{4}\right) \mid w_{1} w_{2}+w_{3} w_{4}=0\right\} \quad \text { be a hyper surface } \\
S_{2}^{3} & =\left\{w=\left(w_{1}, w_{2}, w_{3}, w_{4}\right) \mid w_{1}^{2}-w_{2}^{2}+w_{3}^{2}-w_{4}^{2}=1\right\} \text { be a unit sphere } \\
K & =\left\{w=\left(w_{1}, w_{2}, w_{3}, w_{4}\right) \mid w_{1}^{2}-w_{2}^{2}+w_{3}^{2}-w_{4}^{2}=0\right\} \text { be a null cone in } E_{2}^{4} .
\end{aligned}
$$

Let us consider the following curve:

$$
\begin{aligned}
\alpha & : \quad I \subset R \rightarrow M \subset E_{2}^{4} \text { defined by } \\
\alpha(t) & =\left[w_{1}(t), w_{2}(t), w_{3}(t), w_{4}(t)\right] \text { for every } t \in I .
\end{aligned}
$$

We suppose that $\alpha(t)$ is a differentiable curve of order $r$. The operator $B$, corresponding to $\alpha(t)$ is defined by

$$
B=N(w)=\left[\begin{array}{rrrr}
w_{1} & -w_{2} & -w_{3} & w_{4} \\
w_{2} & w_{1} & -w_{4} & -w_{3} \\
w_{3} & -w_{4} & w_{1} & -w_{2} \\
w_{4} & w_{3} & w_{2} & w_{1}
\end{array}\right]
$$

Let $\alpha(t)$ be a unit velocity curve. The matrix can be represent as

$$
\begin{gathered}
B=h\left[\begin{array}{cccc}
\frac{w_{1}}{h} & -\frac{w_{2}}{h} & -\frac{w_{3}}{h} & \frac{w_{4}}{h} \\
\frac{w_{2}}{h} & \frac{w_{1}}{h} & -\frac{w_{4}}{h} & -\frac{w_{3}}{h} \\
\frac{w_{3}}{h} & -\frac{w_{4}}{h} & \frac{w_{1}}{h} & -\frac{w_{2}}{h} \\
\frac{w_{4}}{h} & \frac{w_{3}}{h} & \frac{w_{2}}{h} & \frac{w_{1}}{h}
\end{array}\right] \\
B=h . A
\end{gathered}
$$

where,

$$
h: I \subset R \rightarrow R, t \rightarrow h(t)=\sqrt{\left|w_{1}^{2}-w_{2}^{2}+w_{3}^{2}-w_{4}^{2}\right|} \text { and } \alpha(t) \neq 0, \alpha(t) \notin K .
$$

Theorem 3.1. Let $\alpha(t) \in S_{2}^{3} \cap M$. In equation $B=h A$, the matrix $B$ is $S O(4,2)$. 
Proof. If $\alpha(t) \in S_{2}^{3}$,where $w_{1}^{2}-w_{2}^{2}+w_{3}^{2}-w_{4}^{2}=1$, using equation (4), in equation $B=h A$, we find $B^{-1}=\varepsilon B \varepsilon$ and $\operatorname{det} A=1$.

Theorem 3.2. In equation $B=h A$, the matrix $A$ in $E_{2}^{4}$ is semiorthogonal matrix.

Proof. Let $\alpha(t) \notin K$, and $w_{1}(t) w_{2}(t)+w_{3}(t) w_{4}(t)=0$. In equation $B=h A$, the matrix $A$ has been shown by $A^{T} \varepsilon A=\varepsilon$. Let the signature matrix, given in [7], be

$$
\varepsilon=\left[\begin{array}{rrrr}
1 & 0 & 0 & 0 \\
0 & -1 & 0 & 0 \\
0 & 0 & 1 & 0 \\
0 & 0 & 0 & -1
\end{array}\right],
$$

where ,the matrix $A$ is semiorthogonal matrix and $\operatorname{det} A=1$.

\section{A Motion with One Parameter}

Let the fixed space and the motinal space be ,respectively, $R_{0}$ and $R$.In this case, one- parametric motion of $R_{0}$ with respect to $R$ will be denoted by $R_{0} / R$. This motion can be expressed by

$$
\left[\begin{array}{c}
X \\
1
\end{array}\right]=\left[\begin{array}{cc}
h A & C \\
0 & 1
\end{array}\right] \cdot\left[\begin{array}{c}
X_{0} \\
1
\end{array}\right]
$$

where, $X$ and $X_{0}$ represent position vectors of any point ,respectively, in $R$ and $R_{0}$, and $C$ represent any translation vector.

Theorem 4.1. The motion defined by the equation in (5) in semi-Euclidean space $E_{2}^{4}$ is a homothetic motion.

Proof. The matrix determined by the equation in (5), can be written as $\mathrm{B}=\mathrm{hA}$, where, due to $A \in S O(4,2)$, this matrix determined is a motion with one parameter.

Theorem 4.2. Let $\alpha(t)$ be a unit velocity curve and $\dot{\alpha}(t) \in M$ then the derivation operator $\dot{B}$ of $B=h A$ is semiorthogonal matrix in $E_{2}^{4}$.

Proof. Since $\alpha(t)$ is unit velocity curve,

$$
\dot{w}_{1}^{2}-\dot{w}_{2}^{2}+\dot{w}_{3}^{2}-\dot{w}_{4}^{2}=1
$$

and $\dot{\alpha}(t) \in M$, then $\dot{w}_{1} \dot{w}_{2}+\dot{w}_{3} \dot{w}_{4}=0$. Thus $\dot{B} \varepsilon \dot{B}^{T}=\dot{B}^{T} \varepsilon \dot{B}$ and $\operatorname{det} \dot{B}=1$. 


\section{Pole Points and Pole Curves of the Motion}

To find the pole point, we have to solve the equation

$$
\dot{B} X+\dot{C}=0
$$

Any solution of equation (6) is a pole point of the motion at that instant in $R_{0}$. Because, by Therom 4 , we have $\operatorname{det} \dot{B}=1$. Hence the equation (6) has only one solution, i.e. $X=\left(-\dot{B}^{-1}\right)(\dot{C})$ at every $t$-instant. In this case the following theorem can be given.

Theorem 5.1. If $\alpha(t)$ is a unit velocity curve and $\dot{\alpha}(t) \in M$, then the pole point corresponding to each $t$-instant in $R_{0}$ is the rotation by $\dot{B}$ of the speed vector $\dot{C}$ of the translation vector at that moment.

Proof. Since the matrix $\dot{B}$ is semiorthogonal, then the matrix $\dot{B}^{T}$ is semiorthogonal , too. Thus it makes a rotation.

Theorem 5.2. Only the pole point corresponding to each t-instant has at the homothetic motion which is defined by the equation in (6) through the space curve in $E_{2}^{4}$.

Proof. Since equation (6) has only one solution at every $t$ - instant,the proof is obvious.

\section{Accelaration Centres of Order $(r-1)$ of a Motion}

Definition 6.1. The set of the zeros of sliding acceleration of order $r$ is called the acceleration centre of order ( $\mathrm{r}-1)$.

By the above definition, we have to find the solutions of the equation

$$
B^{(r)} X+C^{(r)}=0
$$

where

$$
B^{(r)}=\frac{d^{r} B}{d t^{r}} \text { and } C^{(r)}=\frac{d^{r} C}{d t^{r}} .
$$

Let $\alpha(t)$ be a regular curve of order $r$ and $\alpha^{(r)}(t) \in M$. Then we have

$$
w_{1}^{(r)} w_{2}^{(r)}+w_{3}^{(r)} w_{4}^{(r)}=0
$$

Thus,

$$
\left(w_{1}^{(r)}\right)^{2}-\left(w_{2}^{(r)}\right)^{2}+\left(w_{3}^{(r)}\right)^{2}-\left(w_{4}^{(r)}\right)^{2} \neq 0 \quad, w_{i}^{(r)}=\frac{d^{r} w_{i}}{d t^{r}}
$$


Also,we have

$$
\operatorname{det} B^{(r)}=\left[\left(w_{1}^{(r)}\right)^{2}-\left(w_{2}^{(r)}\right)^{2}+\left(w_{3}^{(r)}\right)^{2}-\left(w_{4}^{(r)}\right)^{2}\right]^{2} .
$$

Then $\operatorname{det} B^{(r)} \neq 0$. Therefore the matrix $B^{(r)}$ has an inverse and by the equation in $(7)$, the acceleration centre of order $(r-1)$ at every $t$-instant, is

$$
X=\left[B^{(r)}\right]^{-1}\left[-C^{(r)}\right] .
$$

Example 6.2. Let $\alpha: I \subset R \rightarrow M \subset E_{2}^{4}$ be a curve given by

$$
t \rightarrow \alpha(t)=\frac{1}{\sqrt{2}}(\text { cht }, \text { sht }, \text { cht },-\operatorname{sh} t) .
$$

Note that $\alpha(t) \in S_{2}^{3}$ and since $\|\dot{\alpha}(t)\|=1$, then $\alpha(t)$ is a unit velocity curve.Moreover, $\dot{\alpha}(t) \in M, \ddot{\alpha}(t) \in M, \ldots, \alpha^{(r)}(t) \in M$. Thus $\alpha(t)$ satisfies all conditions of the above theorems.

ÖZET: Bu çalışmada, bikompleks sayılar için [1] de tanımlanan eşlenik tanımlarından bir tanesini ele aldık. Bu eşlenik yardımıyla, $E_{2}^{4}$ semi - Öklidiyen uzayda bir metrik tanımland. Bu metriği kullanarak $E_{2}^{4}$ de bir hareket tanımladık. $E_{2}^{4}$ de bir M hiperyüzeyi üzerindeki bir eğri yardımıyla tanımlanan hareketin homotetik hareket olduğunu gösterdik. Ayrıca, M hiperyüzeyi üzerinde r. mertebeden regüler olan bir eğri yardımıyla tanımlanan hareketin her $\mathrm{t}$ anında (r-1) inci mertebeden bir tek ivme merkezinin olduğu gösterildi.

\section{REFERENCES}

[1] Dominic Rochon and S.Tremblay, Bicomplex Quantum Mechanics: II. The Hilbert Space Adv. appl. Clifford alg. DOI 10.1007/s00006-003-0000, Birkhauser Verlag Basel/Switzerland, (2006)

[2] Dominic Rochon and M. Shapiro, On algebraic properties of bicomplex and hyperbolic numbers,Anal. Univ.Oradea,fasc.math.,vol.11,71-110 (2004).

[3] O.P. Agrawal, Hamilton Operators and Dual Number Quaternions in Spatial Kinematics, Mec-Mach Theory (22),569-575(1987).

[4] H.Kabadayı, Y.Yayl , Homothetic motion at $E^{4}$ with bicomplex numbers, Applied Mathematics Letters (Submitted)

[5] Y. Yayl, Homothetic motions at $E^{4}$, Mech. Mach Theory 27(3), 303-305 (1992).

[6] G.B. Price, An Introduction to Multicomplex Spaces and Functions, Marcel Dekker, Inc: New York. I (1)-44(1) . (1991).

[7] Barrett O'Neill Semi-Riemannian Geometry, Pure and Applied Mathematics, 103 .Academic Pres, Inc. [Harcourt Brace Jovanovich, Publishers] New York. (1983).

Current address: Department of Mathematics, Faculty of Science, University of Ankara, Tandoğan, Ankara, TURKEY

E-mail address: babadagf@science.ankara.edu.tr,yayli@science.ankara.edu.tr,

ekmekci@science.ankara.edu.tr 